\title{
Study of Various Histogram Equalization Techniques
}

\author{
Prasanth C. R. \\ Pursing Mtech in Digital signal processing Department of Electronics College of engineering cherthala
}

\begin{abstract}
Histogram equalization (HE) works well on single channel images for contrast enhancement. However, the technique used is ineffective on multiple channel images. So, it is not suitable for consumer electronic products, where preserving the original brightness is necessary in order not to introduce unnecessary visual deterioration. Bi-histogram equalization (BHE) has been developed and it is analyzed mathematically.BHE separates the input image's histogram into two, based on its mean before equalizing them independently so that it can preserve the original brightness up to certain extends. Recursive Mean-Separate Histogram Equalization (RMSHE) is another technique to provide better and scalable brightness preservation for gray scale and color images. While the separation is done only once in BHE, RMSHE performs the separation recursively based on their respective mean. It is analyzed mathematically that the output images mean brightness will converge to the input images mean brightness as the number of recursive mean separation increases. The recursive nature of RMSHE also allows scalable brightness preservation, which is very useful in consumer electronics. Finally a comparative study was made to analyze all the above methods using gray scale and color images.
\end{abstract}

Keywords: Bi-histogram equalization, histogram equalization, scalable brightness preservation, recursive mean-separate

\section{INTRODUCTION}

Histogram equalization (HE) is a very popular technique for enhancing the contrast of an image. It basic idea lies on mapping the gray levels based on the probability distribution of the input gray levels. It flattens and stretches the dynamics range of the images histogram and resulting in overall contrast improvement, $\mathrm{HE}$ has been applied in various fields such as medical image processing and radar image processing. But, $\mathrm{HE}$ is not commonly used in consumer electronics such as TV because it may significantly change the brightness of an input image and cause undesirable artifacts. In theory, it can be shown that the mean brightness of the histogram equalized image is always the middle gray level regardless of the input mean. This is not a desirable property in some applications where brightness preservation is necessary. Mean preserving Bi-histogram equalization (BHE) has-been proposed to overcome the above mentioned problems.BHE firstly separate the input images histogram into two based on its mean; one having range from minimum gray level to mean and the other ranges from mean to the maximum gray level. Next, it equalizes the two histograms independently. It has been analyzed both mathematically and experimentally. This technique is capable to preserve the original brightness to a certain extends. There are still cases that are not handled well by BHE.These images require higher degree of brightness preservation to avoid annoying artifacts. Therefore, this paper proposes generalization of BHE to overcome such limitation and provide not only better but also scalable brightness preservation. BHE separates the input images histogram into two based on its mean before equalizing them independently. While the separation is done only once in BHE, this paper proposes to perform the separation recursively; separate each new histogram further based on their respective means. It has-been analyzed mathematically that the output images mean brightness would converge to the input images mean brightness as the number of recursive mean separations increases. Besides, its recursive nature also implies scalable preservation, which is very useful in consumer electronic products. The generalization of BHE, namely - Recursive Mean Separate Histogram Equalization (RMSHE) will be presented with mathematical analysis.

\section{TYPICAL HISTOGRAM EQUALIZATION}

Histogram equalization (HE) has been widely used for contrast enhancement of images by uniformly distributing the probability of intensity values. Histogram equalization is widely used for contrast enhancement in a variety of applications due to its simple function and effectiveness. Examples include medical image processing and radar signal processing. One drawback of the histogram equalization can be found on the fact that the brightness of an image can be changed after the histogram equalization, which is mainly due to the flattening property of the histogram equalization.

Let $\mathrm{X}=\{X(I, j)\}$ denote a given image composed of $L$ discrete gray levels denoted as $\left\{\mathrm{X}_{0}, \mathrm{X}_{1, \ldots \ldots \ldots} \mathrm{X}_{\mathrm{L}-1}\right\}$ where $X(i, j)$ represents an intensity of the image at the spatial location $(i, j)$ and $X(i, j) €\left\{\mathrm{X}_{0,}, \mathrm{X}_{1, \ldots \ldots \ldots . . . . . . . . .} \mathrm{X}_{\mathrm{L}-1}\right\}$. For a given image $\mathbf{X}$, the probability density function $\mathrm{p}(\mathrm{Xk})$ is defined as 


$$
p\left(X_{k}\right)=\frac{n^{k}}{n}
$$

for $\boldsymbol{K}=0,1, \ldots, L-1$, where $n^{K}$ represents the number of times that the level $X_{K}$ appears in the input image X and $n$ is the total number of samples in the input image. Note that $p(X k)$ is associated with the histogram of the input image which represents the number of pixels that have a specific intensity $X_{k}$. In fact, a plot of $n^{k}$ vs. $X_{k}$ is known as the histogram of $\mathbf{X}$. Based on the probability density function, we define the cumulative density function as

$$
c(x)=\sum_{j=0}^{k} p\left(X_{j}\right)
$$

Where $\mathrm{k}=\mathrm{O}, 1 \ldots \mathrm{L}-1$, and it is obvious that $\mathrm{c}\left(\mathrm{X}_{\mathrm{L}-1}\right)=1$. Thus the transform function of histogram equalization can be defined as

$$
f(x)=X_{0}+\left(X_{L-1}-X_{0}\right) c(x)
$$

Then the output image of the histogram equalization, $\mathbf{Y}=\{\mathrm{Y}(\mathrm{i}, \mathrm{j})$,$\} , can be expressed as$

$$
\begin{aligned}
& \mathbf{Y}=f(\mathbf{X}) \\
& =\{f(X(i, j) \mid \forall X(i, j) \in \mathbf{X}\}
\end{aligned}
$$

The disadvantages of typical Histogram equalization method is that it cannot however maintain average brightness level which may result in either under or over saturation in the processed image and Overall contrast of the input image is degraded after the histogram equalization. More fundamental reason behind the limitations of the histogram equalization is that the histogram equalization does not take the mean brightness of an image into account. In the subsequent sections, a new contrast enhancement algorithm is formally proposed based on the histogram equalization. The new algorithm utilizes the mean brightness which is the one of the important statistics of an image. This is known as brightness preserving bi histogram equalization (BBHE).

\section{Brightness Preserving Bi Histogram Equalization (BBHE)}

The ultimate goal of the proposed algorithm is to preserve the mean brightness of a given image while the contrast is enhanced. The BBHE firstly decomposes an input image into two sub images based on the mean of the input image. One of the sub images is the set of samples less than or equal to the mean whereas the other one is the samples greater than the mean. Then the BBHEequalizes the sub images independently based on the respective histograms with the constraint that the samples in the formal set are mapped into the range from the minimum gray level to the input mean and the samples in the latter set are mapped into the range from the mean to the maximum gray level. In other words, one of the sub images are equalized over the range up to the mean and the other sub images equalized over the range from the mean based on the respective histograms. Thus, the resulting equalized sub images are bounded by each other around the input mean, which has an effect of preserving mean brightness.

Denote by $\mathrm{Xm}$ the mean of the image $\mathbf{X}$ and assume that $\mathrm{Xm}\left\{\mathrm{X}_{0}, \mathrm{X} 1 \ldots \mathrm{X}_{\mathrm{L}-1}\right\}$. Based on the mean, the input image is decomposed into two sub-images $\mathbf{X}_{\mathrm{L}}$ and $\mathbf{X}_{\mathrm{U}}$ as

$$
\mathbf{X}=\mathbf{X}_{L} \cup \mathbf{X}_{U}
$$

Where

$$
\begin{gathered}
\mathbf{X}_{L}=\left\{X(\mathrm{i}, \mathrm{j}) \mid X(\mathrm{i}, \mathrm{j}) \leq X_{m}, \forall X(\mathrm{i}, \mathrm{j}) \in \mathbf{X}\right\} \\
\mathbf{X}_{U}=\left\{X(\mathrm{i}, \mathrm{j}) \mid X(\mathrm{i}, \mathrm{j})>X_{m}, \forall X(\mathrm{i}, \mathrm{j}) \in \mathbf{X}\right\}
\end{gathered}
$$

Note that the sub-image $\mathbf{X}_{L}$ is composed of $\left\{\mathrm{X}_{0}, \mathrm{X}_{1} \ldots \mathrm{Xm}\right\}$ and the other image $\mathbf{X}_{U}$ is composed of $\{\mathrm{Xm}+1$, $\left.\mathrm{Xm}+2 \ldots \mathrm{X}_{\mathrm{L}-1}\right\}$.Next, define the respective probability density functions of the sub-images $\mathbf{X}_{L}$ and $\mathbf{X}_{U}$ as 


$$
p_{L}\left(X_{k}\right)=\frac{n_{L}^{k}}{n_{L}}
$$

Where $k=0,1 \ldots m$, and

$$
p_{U}\left(X_{k}\right)=\frac{n_{U}^{k}}{n_{U}}
$$

Where $\mathrm{k}=\mathrm{m}+1, \mathrm{~m}+2 \ldots \mathrm{L}-1$, in which $n_{\mathrm{L}}{ }^{\mathrm{k}}$ and $n_{\mathrm{u}}{ }^{\mathrm{k}}$ represen $t$ the respective numbers of $\mathrm{Xk}$ in $\mathbf{X}_{L}$ and $\mathbf{X}_{U}$, and $\mathrm{n}_{\mathrm{L}}$ and $\mathrm{n}_{\mathrm{U}}$ are the total number of samples in $\mathbf{X}_{L}$ and $\mathbf{X}_{U}$ respectively. The respective cumulative density functions for $\mathbf{X}_{L}$ and $\mathbf{X}_{U}$ are then defined as

$$
\begin{aligned}
& c_{L}(x)=\sum_{i=n}^{k} p_{L}\left(X_{j}\right) \\
& c_{U}(x)=\sum_{j=m+1}^{k} p_{U}\left(X_{j}\right)
\end{aligned}
$$

Similar to the case of HE where a cumulative density function is used as a transform function, let's define the following transform functions exploiting the cumulative density functions

$$
\begin{gathered}
f_{L}(x)=X_{0}+\left(X_{m}-X_{0}\right) c_{L}(x) \\
f_{U}(x)=X_{m+1}+\left(X_{L+1}-X_{m+1}\right) c_{U}(x)
\end{gathered}
$$

Based on these transform functions, the decomposed sub images are equalized independently and the composition of the resulting equalized sub-images constitute the output of BBHE.That is, the output image of BBHE, $\mathbf{Y}$, is finally expressed as

$$
\begin{aligned}
\mathbf{Y} & =\{Y(i, j)\} \\
& =f_{L}\left(\mathbf{X}_{L}\right) \cup f_{U}\left(\mathbf{X}_{U}\right),
\end{aligned}
$$

Where

$$
\begin{aligned}
& f_{L}\left(\mathbf{X}_{L}\right)=\left\{f_{L}(X(i, j)) \mid \forall X(i, j) \in \mathbf{X}_{L}\right\} \\
& f_{U}\left(\mathbf{X}_{U}\right)=\left\{f_{U}(X(i, j)) \mid \forall X(i, j) \in \mathbf{X}_{U}\right\}
\end{aligned}
$$

it is easy to see that $f_{L}\left(\mathbf{X}_{L}\right)$ equalizes the sub-image $\mathbf{X}_{\mathrm{L}}$ over the range $\left(\mathrm{X}_{0}, \mathrm{X}\right.$ m) whereas $\mathrm{f}_{\mathrm{U}}(\mathrm{Xu})$ equalizes the sub-image $\mathbf{X U}$ over the range $\left(\mathrm{X} \mathrm{m}+1, \mathrm{X}_{\mathrm{L}-1}\right)$. As a consequence, the input image $\mathbf{X}$ is equalized over the entire dynamic range $\left(\mathrm{X}_{0}, \mathrm{X}_{\mathrm{L}-1}\right)$ with the constraint that the sample less than the input mean are mapped to $\left(\mathrm{X}_{0}, \mathrm{X} \mathrm{m}\right)$ and the samples greater than the mean are mapped to $\left(\mathrm{X} \mathrm{m}+1, \mathrm{X}_{\mathrm{L}-1}\right)$.

\subsection{Analysis on the brightness change by BBHE}

Suppose that $\mathbf{X}$ is a continuous random variable, i.e., $L=\infty$, then the output of the HE, $\mathbf{Y}$ is also regarded as a random variable. It is well known that the HE produces an image, whose gray levels have a uniform density, i.e

$$
p(x)=1 /\left(X_{L-1}+X_{0}\right)
$$

For $\mathrm{X}_{0} \leq \mathrm{x} \leq \mathrm{X}_{\mathrm{L}-1}$. Thus, it is easy to show that the mean brightness of the output image of the HE is the middle gray level since 


$$
\begin{aligned}
& E(Y)=\sum_{X_{0}}^{X_{L-1}} x p(x) d x \\
& =\sum_{X_{0}}^{X_{L-1}} \frac{x}{X_{L-1}-X_{0}} d x \\
& =\frac{X_{L-1}+X_{0}}{2}
\end{aligned}
$$

Where $E$ (.) denotes a statistical expectation. It should be emphasized here that the output mean of the HE has nothing to-do with the input image. That is, it is always the middle gray level no matter how much the input image is bright/dark. Clearly, this property is not desirable in many applications. Turning the attention to the man change by the BBHE, suppose that $\mathbf{X}$ is a random variable, which has symmetric distribution around its mean, Xm. When the sub-images are equalized independently, the mean brightness of the output of the BBHE can be expressed as

$$
\begin{gathered}
E(\mathbf{Y})=E\left(\mathbf{Y} \mid \mathbf{X} \leq X_{m}\right) \operatorname{Pr}\left(\mathbf{X} \leq X_{m}\right)+E\left(\mathbf{Y} \mid \mathbf{X}>X_{m}\right) \operatorname{Pr}\left(\mathbf{X}>X_{m}\right) \\
=1 / 2\left\{E\left(\mathbf{Y} \mid \mathbf{X} \leq X_{m}\right)+E\left(\mathbf{Y} \mid \mathbf{X}>X_{m}\right)\right\}
\end{gathered}
$$

Where $\operatorname{Pr}(\mathbf{X} \leq \square \mathbf{X m})=\operatorname{Pr}(\mathbf{X}>\mathbf{X m})=1 / 2$ is used since $\mathbf{X}$ is assumed to have asymmetric distribution around $\mathrm{Xm}$. From above equations we can write

$$
\begin{aligned}
& E\left(\mathbf{Y} \mid \mathrm{X} \leq X_{m}\right)=\left(X_{0}+X_{m}\right) / 2 \\
& E\left(\mathbf{Y} \mid \mathrm{X}>X_{m}\right)=\left(X_{m}+X_{L-1}\right) / 2
\end{aligned}
$$

Substituting (24) and (25) in equation (23) we get

$$
E(\mathbf{Y})=\left(X_{m}+X_{G}\right) / 2
$$

Where

$$
X_{G}=\left(X_{0}+X_{L-1}\right) / 2
$$

Is the middle gray level, which implies that the mean brightness of the equalized image by BBHE locates in the middle of the input mean and the middle gray level. Note that the output mean of the BBHE is a function of the input mean brightness Xm. This fact clearly indicates that the BBHE preserves the brightness compared to the case of typical HE where output mean is always the middle gray level.

In typical HE, no mean-separation is performed and thus, no brightness preservation. In BBHE, the mean-separation is done once and thus, achieve certain extends of brightness preservation. In cases where more brightness preservation is required, perform the mean separation recursively; separate the resulting histograms again based on their respective means. This is known as Recursive Mean-Separate Histogram Equalization (RMSHE). This is basically a generalization of $\mathrm{HE}$ and BBHE in the aspect of brightness preservation

\section{Recursive Mean Seperparate Histogram Equalization (RMSHE)}

the output mean $\mathrm{E}(\mathbf{Y})$ of typical HE is given as follows:

$$
E(\mathbf{Y})=\left(X_{0}+X_{L-1}\right) / 2=X_{G}
$$

No mean-separation is performed before equalization and hence, no brightness preservation. Where output mean is always the middle gray level, $\mathrm{XG}$ and has nothing to do with input mean. In fact, HE is equivalent to RMSHE with recursion level, $\mathrm{r}=0$.Fig. 4.1 shows histogram before and after BBHE. As the output mean, $E(\mathbf{Y})$ after BBHE is as follows

$$
E(\mathbf{Y})=\left(X_{m}+X_{G}\right) / 2
$$

One mean-separation is performed before equalization and hence, results in some level of brightness preservation. This is indicated by equation (29) where input mean, Xm has equal weight as the middle gray level, $\mathrm{XG}$ in the output mean. In fact, BBHE is equivalent to RMSHE with recursion level $r=1$. In order to 
achieve higher brightness preservation, this paper proposed to perform the mean separation recursively; separate the resulting histograms again based on their respective means. Supposed that $\mathbf{X}$ is further separated into 4 portions based on the mean of the two new histograms, Xml and Xmu as shown in Fig. 4.3

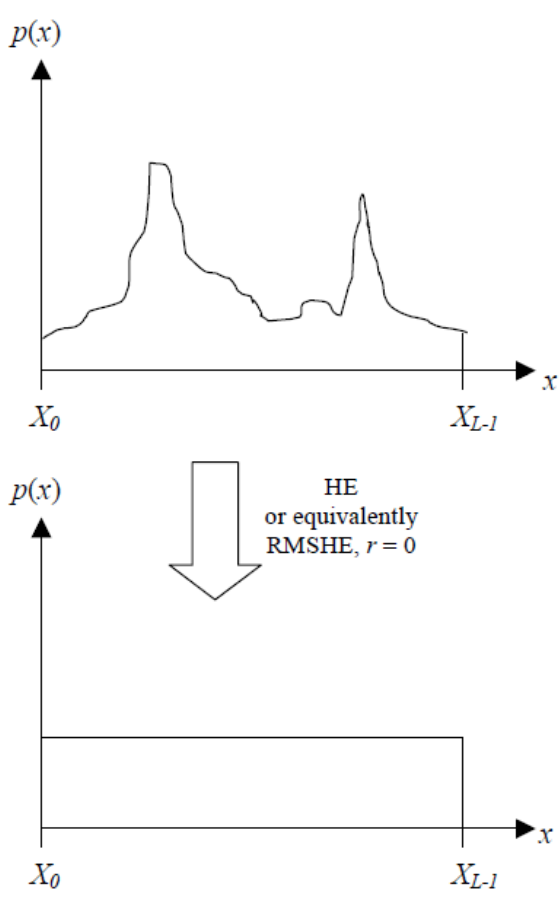

Fig. 4.1 Histogram before and after HE or equivalently,RMSHE, $r=0$

Let's define Xml and Xmu

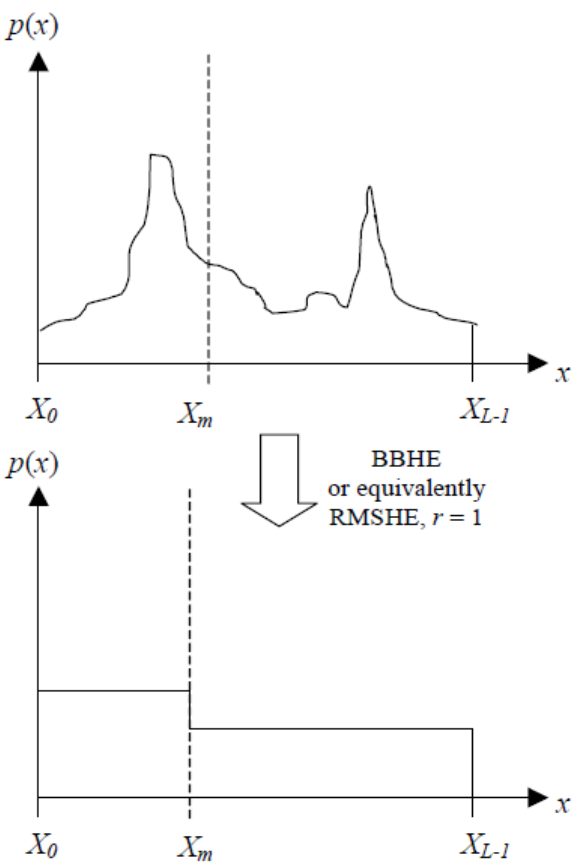

Fig. 4.2 Histogram before and after HE or Equivalently RMSHE.,r=1

$$
\begin{aligned}
& X_{m l}=\frac{\int_{X_{0}}^{X_{m}} x p(x) d x}{\int_{X_{0}}^{X_{m}} p(x) d x}=2 \int_{X_{0}}^{X_{m}} x p(x) d x \\
& X_{m u}=\frac{\int_{X_{m}}^{X_{L-1}} x p(x) d x}{\int_{X_{m}}^{X_{L-1}} p(x) d x}=2 \int_{X_{m}}^{X_{L-1}} x p(x) d x
\end{aligned}
$$

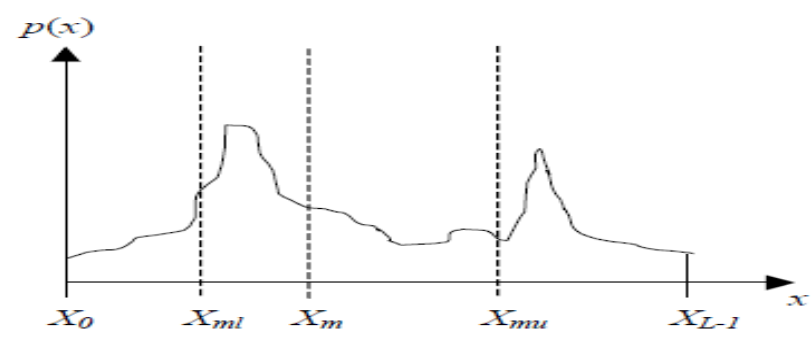

RMSHE, $r=2$

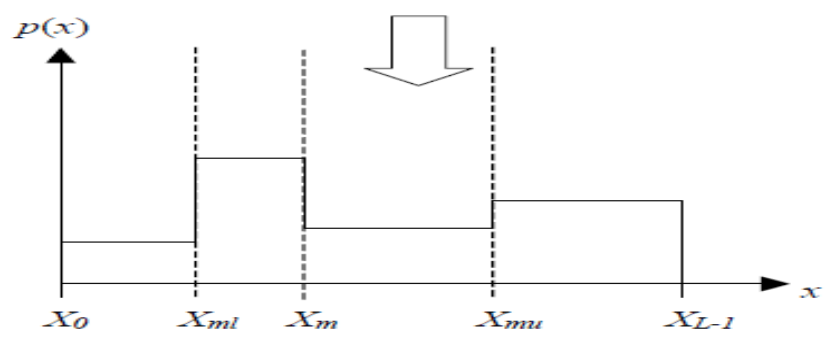

Fig. 4.3 Histogram before and after, RMSHE, $r=2$ 
Because $\mathbf{X}$ is assumed to have a symmetric distribution around $X_{m}$. Fig. 4.3 shows the histogram before and after equalizing the four portions of the histogram independently. This is the result of RMSHE with recursion level, $r$ $=2$. The following shows the formulation of the output mean.

$$
\begin{aligned}
E(\mathbf{Y}) & =E\left(\mathbf{Y} \mid \mathbf{X} \leq X_{m l}\right) \operatorname{Pr}\left(\mathbf{X} \leq X_{m}\right) \\
& +E\left(\mathbf{Y} \mid X_{m l}<\mathbf{X} \leq X_{m}\right) \operatorname{Pr}\left(X_{m l}<\mathbf{X} \leq X_{m}\right) \\
& +E\left(\mathbf{Y} \mid X_{m}<\mathbf{X} \leq X_{m u}\right) \operatorname{Pr}\left(X_{m}<\mathbf{X} \leq X_{m u}\right) \\
+ & E\left(\mathbf{Y} \mid \mathbf{X}>X_{m u}\right) \operatorname{Pr}\left(\mathbf{X}>X_{m u}\right) \\
=1 / 4 & \left\{E\left(\mathbf{Y} \mid \mathbf{X} \leq X_{m l}\right)+E\left(\mathbf{Y} \mid X_{m l}<\mathbf{X} \leq X_{m}\right)\right. \\
& \left.+E\left(\mathbf{Y} \mid X_{m}<\mathbf{X} \leq X_{m u}\right)+E\left(\mathbf{Y} \mid \mathbf{X}>X_{m u}\right)\right\}
\end{aligned}
$$

where $\operatorname{Pr}(\mathbf{X} \leq \square \mathrm{Xml})=\operatorname{Pr}(\mathrm{Xml}<\mathbf{X} \leq \mathrm{Xm})=\operatorname{Pr}(\mathrm{Xm}<\mathbf{X} \leq \square \mathrm{Xmu})=\operatorname{Pr}(\mathbf{X}>\mathrm{Xmu})=1 / 4$ is used since $\mathbf{X}$ is assumed to have a symmetric distribution around $\mathrm{Xm}$

$$
\begin{aligned}
E(\mathbf{Y}) & =1 / 4\left\{\left[\left(X_{0}+X_{m l}\right) / 2\right]+\left[\left(X_{m l}+X_{m}\right) / 2\right]\right. \\
& \left.+\left[\left(X_{m}+X_{m u}\right) / 2\right]+\left[\left(X_{m u}+X_{L-1}\right) / 2\right]\right\} \\
& =1 / 4\left\{\left[\left(X_{0}+X_{L-1}\right) / 2\right]+\left[2\left(X_{m l}+X_{m u}\right) / 2\right]+X_{m}\right\} \\
& =1 / 4\left\{X_{G}+2 X_{m}+X_{m}\right\} \\
& =1 / 4\left\{X_{G}+3 X_{m}\right\}
\end{aligned}
$$

From (29) and (30)

Two mean-separations before equalization results in higher-level of brightness preservation compare to zero and one mean-separations as in the case of HE and BBHE respectively. This is indicated by equation (33) where the weight of input mean, $\mathrm{Xm}$ has increased to three times as much as the weight of middle gray level, $\mathrm{X}_{\mathrm{G}}$. Following the above discussion, it is very reasonable to expect that the brightness preservation will increase as the number of recursive mean-separations increases. Base on the observations on the output mean for RMSHE recursion level $r=0,1$ and 2 shown above, it is not difficult to generalize the output mean E (Y) for RMSHE recursion level $\mathrm{r}=\mathrm{n}$ as follows:

$$
\begin{aligned}
r & =0, E(\mathbf{Y})=X_{G} \\
r & =1, E(\mathbf{Y})=\left(X_{m}+X_{G}\right) / 2 \\
r & =2, E(\mathbf{Y})=\left(3 X_{m}+X_{G}\right) / 4 \\
. & \\
r & =n, E(\mathbf{Y})=\left(\left(2^{n}-1\right) X_{m}+X_{G}\right) / 2^{n} \\
& =X_{m}+\left[\left(X_{G}-X_{m}\right) / 2^{n}\right]
\end{aligned}
$$

Equation indicates that as the recursion level, $\mathrm{n}$ grows larger, $\mathrm{E}(\mathbf{Y})$ will eventually converge to the input mean, Xm.In other words, it allows scalable degree of brightness preservation range from $0 \%$ (output of $\mathrm{HE}$ ) - $100 \%$ (getting back the original image). In applications of consumer electronics, the variety of image involve are too wide to be covered with only a specific level of brightness preservation. Therefore, the scalability in this algorithm is the most desirable property to allow adjustment of level of brightness preservation base on individual image's requirement. Equation indicates that as the recursion level, $\mathrm{n}$ grows larger, $\mathrm{E}(\mathbf{Y})$ will eventually converge to the input mean, Xm.In other words, it allows scalable degree of brightness preservation range from $0 \%$ (output of $\mathrm{HE}$ ) - $100 \%$ (getting back the original image). In applications of consumer electronics, the variety of image involve are too wide to be covered with only a specific level of brightness preservation. Therefore, the scalability in this algorithm is the most desirable property to allow adjustment of level of brightness preservation base on individual image's requirement 


\section{Conclusion}

In this paper, a new contrast enhancement algorithm referred as the recursive mean-separate histogram equalization (RMSHE) with scalable brightness preservation is proposed. The RMSHE is a generalization of HE and BHE in term of brightness preservation. The main idea lies on recursively separating the input histogram based on the mean. The ultimate goal behind in the RMSHE is to allow higher-level of brightness preservation to avoid unwanted noises. The analysis shows that the output mean will converge into the input mean as the number of recursive mean-separation increases. Therefore, the scalability in this algorithm is the most desirable property especially in consumer electronics to allow scaling of brightness preservation, suited to individual image.

\section{REFERENCES}

[1] R. Gonzalez and R. Woods, Digital Image Processing, 3rd ed. Englewood Cliffs, NJ: Prentice-Hall, 2007

[2] Y. Kim, "Contrast enhancement using brightness preserving bi-histogram Equalization," IEEE Transactions

[3] S. Chen and A. Ramli, "Contrast enhancement using recursive mean separate Histogram equalization for scalable brightness preservation, IEEE Transactions

\section{AUTHORS PROFILE}

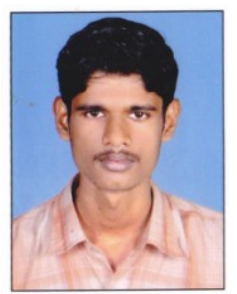

PrasanthC.R Received Diploma in Electronics and Instrumentation from govt. Polytechnic cherthala (first class with distinction, third rank and gold medallist). He passed Btech in Electronics and Communication Engg from M.G university kerala, india.he currently pursing Mtech in Digital signal processing from Govt. Engg college cherthala under Cochin University of science and technology 